\title{
Micelle Formation in Myristyltrimethyl Ammonium Bromide at Different Solvent Composition of Ethanol Water Mixed Solvent Media.
}

\author{
Prem Kumar Shrestha ${ }^{1}$, Jaya Narayan Mitruka ${ }^{2}$ and Subash Kumar Chapagain ${ }^{2 *}$ \\ ${ }^{1}$ Padma Kanya Multiple Campus, Tribhuvan University, Kathmandu \\ ${ }^{2}$ Mechi Multiple Campus, Bhadratpur, Nepal \\ Email: subaschapagain@gmail.com
}

\begin{abstract}
pH Changes as a function of concentration for myristyltrimethyl ammonium bromide in pure water and ethanol-mixed solvent media containing 0.1 and 0.2 volume fraction of ethanol at room temperature were observed by the addition of $1 \mathrm{~N} \mathrm{HCl}$ and $1 \mathrm{~N} \mathrm{KOH}$. The critical micelle concentration (CMC) for the myristyltrimethyl ammonium bromide /HCI/KOH system increases when the amount of ethanol increases In water.
\end{abstract}

Keywords: Myristyltrimethyl ammonium bromide (MTABr), $\mathrm{HCl}, \mathrm{KOH}$, ethanol, water digital pH meter.

\section{Introduction}

In chemistry, surfactant is a species that accoumulates at the interface of two phases or substances and modifies the properties of the surface. Many surfactants play an important role in the field of science and technology due to their unique properties ${ }^{1,2}$. In an aqueous medium, both pure and mixed surfactants form micelles after reaching a concentration called the critical micelle concentration (CMC), whose determination has considerable practical importance normally to understand the self-organizing behaviors of surfactants in exact and detailed ways. Many studies have been devoted to the elucidation of micelle structures under different conditions ${ }^{2-4}$. There are so many articles for CMC calculation from conductivity measurements. But there is less work on $\mathrm{pH}$ of surfactant for CMC calculation. Hence, this research work will be very relevance in the field of research ${ }^{5,6}$. $\mathrm{pH}$ change with mixed-solvent composition may thus reflect the change in solvent structure and ion-solvent interactions ${ }^{7}$. A binary mixture of water and ethanol with ratio varying in a wide range, is the most frequently investigated medium.

\section{Experimental Methods}

The effect of $\mathrm{pH}$ on Myristyltrimethyl ammonium bromide concentration was determined at room temperature with a digital $\mathrm{pH}$ meter at different solvent composition of ethanol-water mixed solvent media. First, $7 \mathrm{mM}$ myristyltrimethyl ammonium bromide solution was prepared, separately, and then each stock solution was diluted with water from 7 to $1 \mathrm{mM}$. For the Myristyltrimethyl ammonium bromide $/ \mathrm{HCl}$ systems, a solution of $7 \mathrm{ml}$ of $7 \mathrm{mM}$ myristyltrimethyl ammonium bromide and $0.05 \mathrm{~mL}$ of $1 \mathrm{~N} \mathrm{HC1}$ and $0.5 \mathrm{ml}$ of $1 \mathrm{~N} \mathrm{KOH}$ was subjected to $\mathrm{pH}$ measurement at room temperature to prevent hydrolysis. For the Myristyltrimethyl ammonium bromide / $\mathrm{KOH}$ systems, a solution of $7 \mathrm{~mL}$ of $7 \mathrm{mM}$ myristyltrimethyl ammonium brodide and $0.05 \mathrm{~mL}$ of $1 \mathrm{~N} \mathrm{KOH}$ and $0.5 \mathrm{ml}$ of $1 \mathrm{~N} \mathrm{HCl}$ was subjected to

${ }^{*}$ Corresponding author 
$\mathrm{pH}$ measurement at room temperature to prevent hydrolysis and at different solvent composition of ethanol water mixed solvent media.

\section{Results and Discussion}

The plot of $\mathrm{pH}$ as a function of surfactant concentration of the Myristyltrimethyl ammonium bromide $/ \mathrm{HCl}$ system in water and in two different ethanol-water mixtures (containing 0.10 and 0.20 volume fraction of ethanol) at room temperature are depicted in figs 2,3 and 4 . The variation of $\mathrm{pH}$ as a function of surfactant concentration of the Myristyltrimethyl ammonium brodide/ $\mathrm{HCl}$ system in water is determined, it is found that the increase of the concentration of the surfactant, the $\mathrm{pH}$ values also increases and then decreases; the breaking point is known as critical micelle concentration (CMC) and found to be $4.52 \mathrm{mM}$. The similar trends has been observed in the variation of $\mathrm{pH}$ as a function of surfactant concentration of the Myristyltrimethyl ammonium brodide/ $\mathrm{HCl}$ system in 0.10 to 0.20 volume fraction of ethanol in water, the CMC is found to be $4.7 \mathrm{mM}$ and $4.80 \mathrm{mM}$ respectively.

The plot of $\mathrm{pH}$ as a function of surfactant concentrationof the Myristyltrimethyl ammonium brodide/KOH system in water and in two different ethanol-water mixtures (containing 0.10 to 0.20 volume fraction of ethanol) at room temperature are depicted in Figs 5, 6 and 7. The variation of $\mathrm{pH}$ as a function of surfactant concentrationof the Myristyltrimethyl ammonium bromide/ $\mathrm{KOH}$ system in water is determined and it is found that the increase of the concentration of the surfactant, the $\mathrm{pH}$ values also increases and then decreases and increases, the breaking point is known as critical micelle concentration $(\mathrm{CMC})$ and found to be $4.39 \mathrm{mM}$. The similar trends has been observed in the variation of $\mathrm{pH}$ as a function of surfactant concentration of the Myristyltrimethyl ammonium bromide/KOH system in 0.10 to 0.20 volume fraction of ethanol in water, the CMC is found to be $4.65 \mathrm{mM}$ and $4.80 \mathrm{mM}$ respectively.

From these figures (1-6), it is evident that CMC increases with decreasing dielectric constant of ethanol water mixed solvent media (Table 1). Our CMC data for pure water of Myristyltrimethyl ammonium bromide match with the literature ${ }^{6}$.

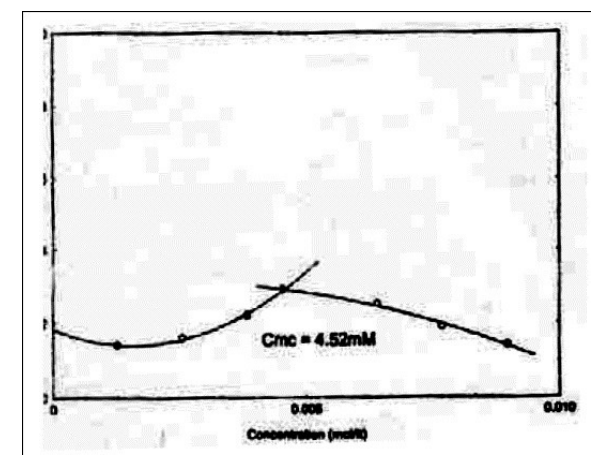

Figure 1: Variationof $\mathrm{pH}$ as a function of surfactant concentration of the Myristyltrimethyl ammonium bromide/ $\mathrm{HCl}$ system in water. 
J. Nepal Chem. Soc., Vol. 37, 2017

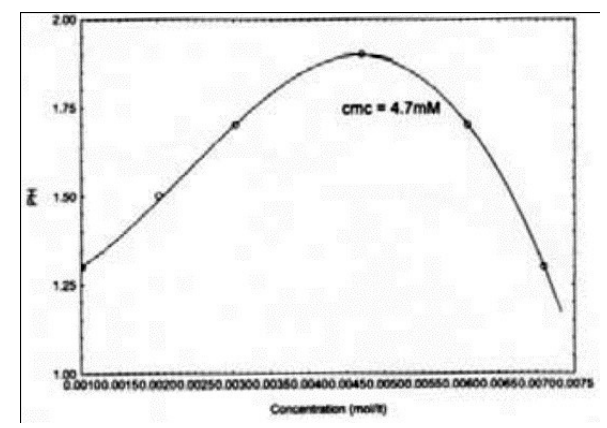

Figure 2: Variation of $\mathrm{pH}$ as a function of surfactant concentration of the Myristyltrimethyl ammonium bromide/ $\mathrm{HCl}$ system in 0.1 volume fraction of ethanol in water.

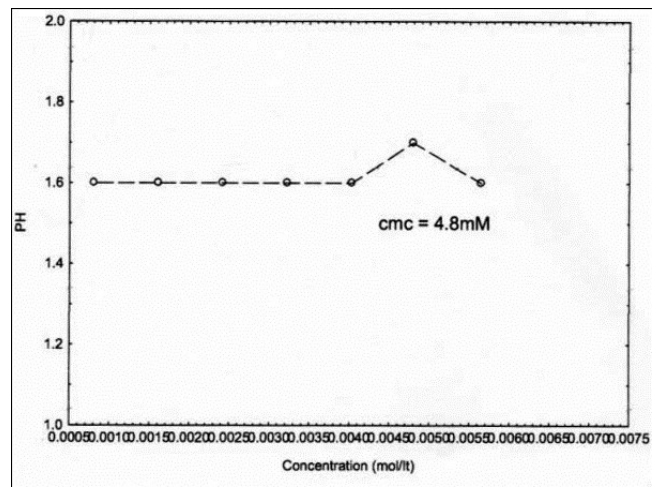

Figure 3: Variation of pH as a function of surfactant concentrationof the Myristyltrimethyl ammonium bromide/HCl system in 0.2 volume fraction of ethanol in water.

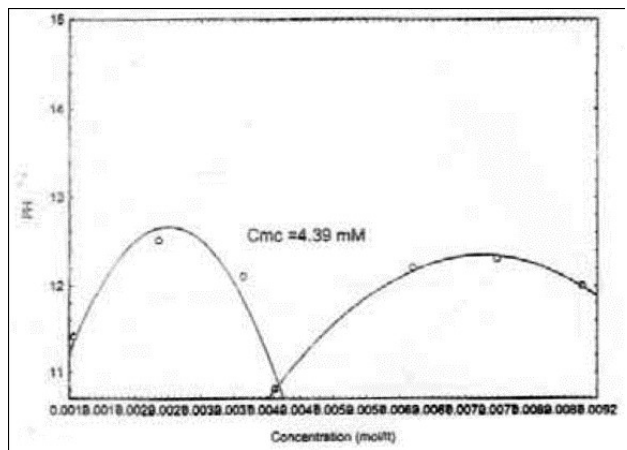

Figure 4: Variation of pH as a function of surfactant concentration of the Myristyltrimethyl ammonium bromide/KOH system in water. 


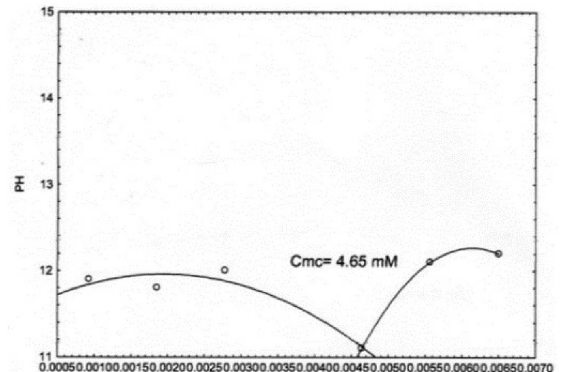

Figure 5: Variation of $\mathrm{pH}$ as a function of surfactant concentration of the Myristyltrimethyl ammonium bromide/ $\mathrm{KOH}$ system in 0.1 volume fraction of ethanol in water.

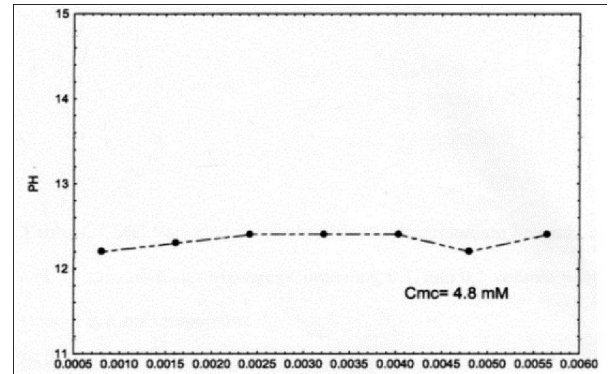

Figure 6: Variation of $\mathrm{pH}$ as a function of surfactant concentration of the Myristyltrimethyl ammonium bromide/KOH system in 0.2 volume fraction of ethanol in water.

\section{Conclusions}

Experimental measurements for $\mathrm{pH}$ of the different concentrations of Myristyltrimethyl ammonium bromide $/ \mathrm{HCl}$ and $\mathrm{KOH}$ systems in pure water and 0.10 and 0.20 volume fractions of ethanol in ethanol (1) + water (2) mixed solvent media have been studied by $\mathrm{pH}$ meter. The following conclusions have been drawn from the above results and discussion:

i) Experimental results for the $\mathrm{pH}$ of solution of Myristyltrimethyl ammonium bromide/HCI and $\mathrm{KOH}$ systems in pure water and 0.10 and 0.20 volume fractions of ethanol in ethanol-water mixed solvent media have been presented as a function surfactant concentration.

ii) The calculated $\mathrm{CMC}$ by the breaking point for Myristyltrimethyl ammonium bromide/ $\mathrm{HCl}$ and $\mathrm{KOH}$ systems are found to be almost same but the $\mathrm{CMC}$ values are found to increase with decreasing dielectric constant of solvent composition.

\section{Acknowledgements}

This work was supported by Research Grant for the fiscal year 2013/2014 from the office of the deam, Institute of Science and Technology, T.U., Kirtipur Nepal under the Research Project scheme. We would like to give thanks to the office of the Deam, Institute of Science and Technology, T.U., Kirtipur Nepal for providing us the golden opportunity in the field of research.

\section{References}

1) R. Shelton, J. Am. Chem. Soc. 1946, 68,753.

2) P. Mukeijee, K.J. Mysells, Natl. Stand. Ref. Data Ser. Nat. Bur. Stand, 1971, 36, 105

3) C. Gonzalez, Appl. Surf. Sci. 2005, 243, 10.

4) Y.H. Li, B.X. Huang, X.Q. Shan, Anal. Bioanal. Chem., 2003, 375, 775.

5) Fuchs-Godec, R. Electrochim. Acta, 2009, 54, 9.

6) A. Quan, A.B. McGeachie, D.J. Keating, E.M. van Dam, J. Rusak, N. Chau, C.S. Malladi, C. Chen, A. McCluskey, M.A. Cousin, P.J. Robinson, Mol. Pharmacol., 2007, 72, 1425.

7) C. Mihali, G. Oprea, \& E. Cical, POLTEHNICA Univ. (Timisoare), 2008, 53, 159.

8) Y. Bayrak, Turk. J. Chem., 2003, 27,487.

9) S. Das, S. Mondal, S. Ghosh, J. Chem. Eng. Data, 2013, 58, 2586.

10) B.D. Flockhart, J. Coll. Sci., 1957, 12,557. 